\title{
Atmospheric Boundary-Layer Dynamics with Constant Bowen Ratio
}

\author{
Amilcare Porporato
}

Received: 21 January 2009 / Accepted: 9 June 2009 / Published online: 26 June 2009

(C) Springer Science+Business Media B.V. 2009

\begin{abstract}
Motivated by the observation that the diurnal evolution of sensible and latent heat fluxes tends to maintain a constant Bowen ratio, we derive approximate solutions of the ordinary differential equations of a simplified atmospheric boundary-layer (ABL) model. Neglecting the early morning transition, the potential temperature and specific humidity of the mixed layer are found to be linearly related to the ABL height. Similar behaviour is followed by the inversion strengths of temperature and humidity at the top of the ABL. The potential temperature of the mixed layer depends on the entrainment parameter and the free-atmosphere temperature lapse rate, while the specific humidity also depends on the free-atmosphere humidity lapse rate and the Bowen ratio. The temporal dynamics appear only implicitly in the evolution of the height of the boundary layer, which in turn depends on the time-integrated surface sensible heat flux. Studying the limiting behaviour of the Bowen ratio for very low and very large values of net available energy, we also show how the tendency to maintain constant Bowen ratio during midday hours stems from its relative insensitivity to the atmospheric conditions for large values of net available energy. The analytical expression for the diurnal evolution of the ABL obtained with constant Bowen ratio is simple and provides a benchmark for the results of more complex models.
\end{abstract}

Keywords Atmospheric boundary layer · Bowen ratio · Evaporation · Mixed layer · Slab model

\section{Introduction}

Mixed-layer or slab models of the atmospheric boundary layer (ABL) have long been used to capture the essential coupling of the land-atmosphere water and energy balances, and the

\footnotetext{
A. Porporato $(\varangle)$

Department of Civil and Environmental Engineering, Duke University, Durham, NC, USA

e-mail: amilcare@duke.edu
}

\author{
A. Porporato \\ EFLUM-EPFL, Lausanne, Switzerland
}


partitioning of incoming solar energy into sensible and latent heat fluxes. This coupling arises because such fluxes depend on the state of the ABL, namely temperature and humidity, which in turn are controlled by the surface fluxes.

The mixed-layer models have been pioneered by Betts (1973), Tennekes (1973) and Carson (1973), and further developed by Tennekes and Driedonks (1981), Driedonks and Tennekes (1984) and many others, to analyze the growth of the ABL and the dynamics of the inversion at its top. These models were then coupled to the evaporation and ABL moisture balance equations to elucidate the role of vegetation and surface conditions on the lower atmosphere and the related feedbacks, as well as used as interfaces between land and atmosphere in climate and biogeochemistry models (de Bruin 1983; McNaughton and Spriggs 1986; Brutsaert 1987; Raupach 1991, 2000; Ek and Mahrt 1994; Lhomme et al. 1998; Daly et al. 2004; Ek and Holtslag 2004; Juang et al. 2007; Siqueira et al. 2009).

After the popularity of slab models in 1980s and 1990s, research has progressively moved towards more complex approaches (Raupach 1998), aimed at more refined descriptions of turbulence, radiation, cloud and surface conditions. However, while such a direction is certainly important to improve predictions and better understand the numerous land-atmosphere feedbacks, simple models should not be dismissed too easily especially when physical interpretation, more than precise forecasting, is the main goal. No matter how refined, in fact, complex models will always miss some high-dimensional components (which instead could be approximated by suitable stochastic components coupled to simple deterministic models), while the essential physics may be obscured by the complexity of the equations and lack of generality of their numerical solutions.

With this in mind, in this paper we take the opposite direction and follow the line of McNaughton and Spriggs (1986) and Raupach (1991) among others, working analytically when possible, and making simplifying approximations to focus on the main processes and key interactions of the problem. Our main line of attack is suggested by field observations whereby sensible and latent heat fluxes tend to follow the diurnal trend of available energy (Brutsaert 2005), giving rise to a roughly constant value of the Bowen ratio, at least during midday hours. While this general tendency is present in both simple and complex models, its physical and mathematical consequences only become apparent when the model is reduced to its essentials. As will be seen, when this approximation is embedded in the dynamical system of the mixed layer, the coupled nonlinear ordinary differential equations become quite simple (linear, in fact), once the effects of the initial conditions (the so-called morning transition; Tennekes 1973) become negligible.

\section{Basic Equations}

The main equations of the mixed layer models are discussed in detail in, e.g., Tennekes (1973), Tennekes and Driedonks (1981), McNaughton and Spriggs (1986), Garratt (1992), and Raupach (2000). These typically consider idealized conditions, neglecting cloud, condensation, radiation and subsidence effects. Here we also assume fixed surface conditions (apart from temperature) during the day, and neglect the effects of the early morning transition, when the nighttime residual layer is dissipated and the ABL grows mostly by mechanical turbulence. As discussed in Tennekes (1973) during the warm season this morning transition is usually less than one hour. All these approximations are quite standard in the literature.

At the surface, the available solar energy is partitioned into latent (evapotranspiration) and sensible heat flux according to 


$$
Q=\lambda E+H,
$$

where $Q$ is the net radiation, $E$ is the evaporative flux, $\lambda$ is the latent heat of vaporization, $H$ is the sensible heat flux. Net radiation is simply assumed to be a function of time $t$, independent of ABL conditions, i.e., $Q(t)$. The previous equation is often written using the Bowen ratio (Bowen 1926; Brutsaert 2005)

$$
Q=\lambda E+H=\lambda E(1+\mathrm{Bo}),
$$

where

$$
\mathrm{Bo}=\frac{H}{\lambda E}
$$

Evapotranspiration is, as usual,

$$
E=g_{e} \rho\left(q_{s}^{*}-q\right)
$$

where $g_{e}$ is the conductance to water vapour (typically the series of stomatal, canopy and atmospheric conductances), $\rho$ is the air density, $q_{s}^{*}$ is the saturated specific humidity at the evaporating surface, and $q$ is the specific humidity of the mixed layer. The saturated specific humidity is a function of the potential temperature $\vartheta_{s}$, according to the Clausis-Clapeyron equation. The sensible heat flux is

$$
H=g_{h} \rho c_{p}\left(\vartheta_{s}-\vartheta\right)
$$

where $g_{h}$ is the conductance to sensible heat transfer (the series of canopy and atmospheric conductances), $c_{p}$ is the constant-pressure specific heat of air, and $\vartheta$ is the potential temperature of the mixed layer.

The previous relationships define the fluxes through the surface layer and into the well-mixed layer, where the specific humidity and potential temperature are constant. The mixed layer grows by turbulent entrainment into the free atmosphere, where the variables are indicated with an ' $f$ ' subscript. In the mixed layer, temperature and humidity are governed by the budget equations for enthalpy and moisture. For potential temperature, the first law of thermodynamics (or enthalpy equation for the conservation of potential temperature) gives

$$
\rho c_{p} h \frac{d \vartheta}{d t}=H+\rho c_{p}\left(\vartheta_{f}-\vartheta\right) \frac{d h}{d t},
$$

where $\gamma_{\vartheta}$ is the potential temperature lapse rate of the free atmosphere, $h$ is the height of the ABL (Fig. 1), and $\vartheta_{f}$ is the potential temperature of the free atmosphere at height $z=h$. The inversion layer at the top of the ABL is assumed to be of negligible depth, as usual in the zeroth-order slab models (Garratt 1992).

The water balance equation in the mixed layer is

$$
\rho h \frac{d q}{d t}=E+\rho\left(q_{f}-q\right) \frac{d h}{d t},
$$

where $q_{f}$ is the specific humidity of the free atmosphere at height $z=h$. Potential temperature and specific humidity in the free atmosphere are assumed to have linear profiles,

$$
\vartheta_{f}=\vartheta_{f_{0}}+\gamma_{\vartheta} h,
$$

and

$$
q_{f}=q_{f_{0}}+\gamma_{q} h .
$$



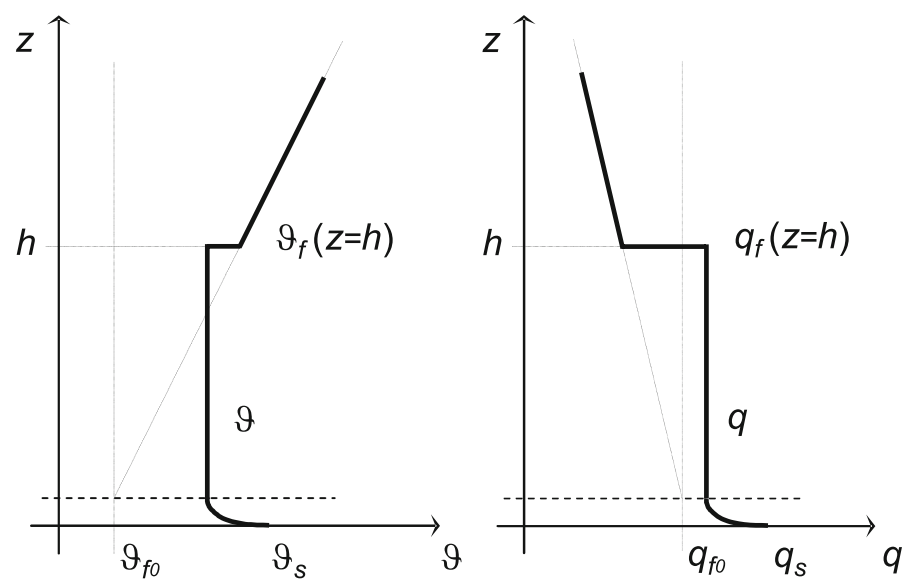

Fig. 1 Schematic representation of the vertical profiles of potential temperature and specific humidity for the mixed-layer ABL model

The ABL grows because of turbulence entrainment at the expense of the mixed-layer turbulent energy (Tennekes 1973; Garratt 1992). A simplified equation for the rate of growth of $h$ can be obtained, as described in the Appendix, as

$$
\frac{d h}{d t}=\frac{(1+2 \beta) H}{\rho c_{p} \gamma_{\vartheta} h}
$$

where the virtual temperature flux, which more precisely should be used to include the effect of water vapour in the buoyancy generation of turbulence (e.g., McNaughton and Spriggs 1986), is approximated by the sensible heat flux, $H_{v} \approx H$. This approximation is quite acceptable, and is comparable to the other approximations made in constructing the mixedlayer models. With $\beta=0$ the equation becomes equivalent to the simple growth model used in McNaughton and Spriggs (1986).

The previous equations form a closed system once $Q(t)$, the surface conditions, through $g_{h}$ and $g_{e}$, and the free atmosphere profiles, (8) and (9), are given.

\section{Analytical Developments}

\subsection{Temperature-Height Relation}

Substituting (10) into (6),

$$
\rho c_{p} h \frac{d \vartheta}{d t}=H\left[1+\frac{(1+2 \beta)\left(\vartheta_{f}-\vartheta\right)}{\gamma_{\vartheta} h}\right],
$$

and using the free atmosphere temperature profile (8) with the notation $\tilde{\vartheta}=\vartheta-\vartheta_{f_{0}}$ yields

$$
\rho c_{p} h \frac{d \tilde{\vartheta}}{d t}=H\left[2(1+\beta)-\frac{(1+2 \beta)}{\gamma_{\vartheta}} \frac{\tilde{\vartheta}}{h}\right] .
$$



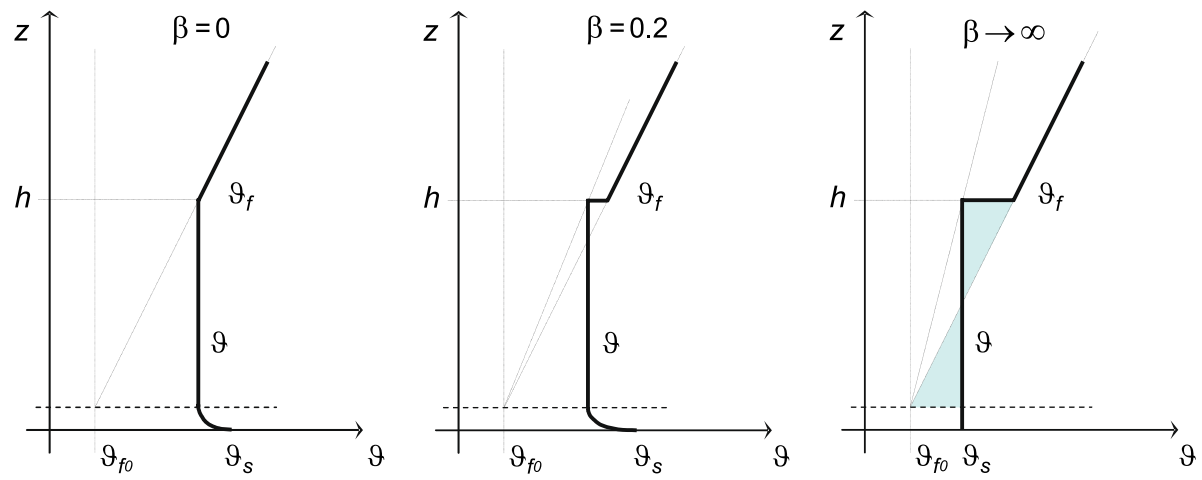

Fig. 2 Types of potential temperature profiles for three different values of the entrainment parameter $\beta$

Dividing then (12) by (10), a single equation can be obtained where time only appears implicitly as a parameter,

$$
\frac{d \tilde{\vartheta}}{d h}=2 \gamma_{\vartheta} \frac{1+\beta}{1+2 \beta}-\frac{\tilde{\vartheta}}{h} .
$$

The solution of (13) is

$$
\tilde{\vartheta}=\gamma_{\vartheta} \frac{1+\beta}{1+2 \beta} h+\frac{C_{1}}{h}
$$

where the integration constant $C_{1}$ can be set equal to zero in order to have $\tilde{\vartheta}=0$ for $h=0$ (consistently with neglecting the morning transient). This results in a linear relationship between the height and the potential temperature of the mixed layer,

$$
\tilde{\vartheta}=\gamma_{\vartheta} \frac{1+\beta}{1+2 \beta} h
$$

previously obtained also by Driedonks (1982), and in agreement with observations, at least before clouds and subsidence become important (Brutsaert, personal communication, 2008).

For $\beta=0$, which corresponds to the case of McNaughton and Spriggs (1986), $\vartheta$ in the $\mathrm{ABL}$ follows the linear profile of the overlying free atmosphere, and no temperature inversion is present. This is the case of pure encroachment, without turbulence entrainment (Garratt 1992). With the more realistic value of $\beta=0.2, \vartheta \approx 0.86 \gamma_{\vartheta} h$, a visible inversion appears, while the ABL grows faster because turbulence entrains air with higher potential temperature from above. Finally, when there is no sensible heat flux at the surface, $H=0$ (which corresponds to $\beta \rightarrow \infty$ ), $\vartheta=1 / 2 \gamma_{\vartheta} h$ and the ABL simply mixes up the enthalpy entrained mechanically from above (Tennekes and Driedonks 1981; Garratt 1992). Figure 2 shows schematically the various cases. As will be seen later, a similar situation holds for the approximate solution of the humidity profiles.

For later use, we also note that introducing (15) into (12) yields

$$
\rho c_{p} h \frac{d \tilde{\vartheta}}{d t}=H(1+\beta)
$$




\subsection{Specific Humidity-Temperature Relation}

A similar procedure can be followed to obtain a relation between specific humidity and temperature. Using the definition of the Bowen ratio (3) and the linear humidity profile (9), and substituting (10) into (7), one obtains

$$
\rho h \frac{d q}{d t}=H\left[\frac{1}{\lambda \mathrm{Bo}}+\frac{1+2 \beta}{c_{p}} \frac{\gamma_{q}}{\gamma_{\vartheta}}+\frac{(1+2 \beta)\left(q_{f_{0}}-q\right)}{c_{p} \gamma_{\vartheta} h}\right] .
$$

Introducing the notation $\tilde{q}=q-q_{f_{0}}$ and the temperature-height relation (15) yields

$$
\rho h \frac{d \tilde{q}}{d t}=H\left[\frac{1}{\lambda \mathrm{Bo}}+\frac{1+2 \beta}{c_{p}} \frac{\gamma_{q}}{\gamma_{\vartheta}}-\frac{1+\beta}{c_{p}} \frac{\tilde{q}}{\tilde{\vartheta}}\right] .
$$

Using (4) and (5), the Bowen ratio can be written as

$$
\mathrm{Bo}=\frac{g_{h} c_{p}\left(\vartheta_{s}-\vartheta\right)}{g_{e} \lambda\left(q_{s}^{*}-q\right)}=\frac{g_{h} c_{p}\left(\tilde{\vartheta}_{s}-\tilde{\vartheta}\right)}{g_{e} \lambda\left(\tilde{q}_{s}^{*}-\tilde{q}\right)},
$$

so that

$$
c_{p} \rho h \frac{d \tilde{q}}{d t}=H\left[\frac{g_{e}\left(\tilde{q}_{s}^{*}-\tilde{q}\right)}{g_{h}\left(\tilde{\theta}_{s}-\tilde{\theta}\right)}+(1+2 \beta) \frac{\gamma_{q}}{\gamma_{\vartheta}}-(1+\beta) \frac{\tilde{q}}{\tilde{\vartheta}}\right] .
$$

Finally, dividing (20) by (16), the sensible heat flux can be eliminated along with the explicit temporal dependence to obtain

$$
\frac{d \tilde{q}}{d \tilde{\vartheta}}=\frac{g_{e} / g_{h}\left(\tilde{q}_{s}^{*}-\tilde{q}\right)}{(1+\beta)\left(\tilde{\vartheta}_{s}-\tilde{\vartheta}\right)}+\frac{(1+2 \beta) \gamma_{q}}{(1+\beta) \gamma_{\vartheta}}-\frac{\tilde{q}}{\tilde{\vartheta}} .
$$

Since $\tilde{q}_{s}^{*}=\tilde{q}_{s}^{*}\left(\tilde{\vartheta}_{s}\right)$, and because $\tilde{\vartheta}_{s}$ is a function of $\tilde{\vartheta}, \tilde{q}$ and $Q(t)$ (due to (1), (4) and (5)), it follows that there is a unique relation between $\tilde{q}$ and $\tilde{\vartheta}$ of the mixed layer. The point of the solution of (21) that is selected on this curve is determined by the diurnal development of the ABL through its height $h$. The ratio $g_{e} / g_{h}$ strongly depends on surface conditions (conductance) and, as we discuss later, it is zero in the dry-surface limit (Bo $\rightarrow \infty)$. It should also be noted that the application of (21) may become problematic when stratification becomes weak.

\section{Solution with Constant Bowen Ratio}

Although we have reduced the problem of relating $\tilde{\vartheta}$ and $\tilde{q}$ to a single first-order ordinary differential equation, the problem remains rather involved due to the nonlinear dependence of both $\tilde{\vartheta}_{s}$ and $\tilde{q}_{s}$ on $\tilde{\vartheta}$ and $\tilde{q}$ through the surface flux equations (4) and (5), and the nonlinear solution of the Clausius-Clapeyron equation for the saturated vapour pressure (and thus the saturated specific humidity) as a function of temperature. Due to these nonlinearities and interdependencies, only a numerical solution and, perhaps, some asymptotic analyses of the full problem seem to be possible. Thus, in what follows we will analyse the problem by making some suitable simplifications.

A drastic but revealing simplification is suggested by the fact that the Bowen ratio tends to remain constant, at least in the central part of the day and for well-watered conditions (i.e., potential evapotranspiration), e.g., Brutsaert and Sugita (1992) and Brutsaert and Chen (1996) [see also Stewart and Thom (1973) and Brutsaert (2005, pp. 139-141)]. For example, 
Table 1 Typical values of the parameters used in computations

\begin{tabular}{lll}
\hline$\rho$ & 1.29 & $\mathrm{~kg} \mathrm{~m}^{-3}$ \\
$\lambda$ & $2.45 \times 10^{6}$ & $\mathrm{~J} \mathrm{~kg}^{-1}$ \\
$c_{p}$ & 1,005 & $\mathrm{~J} \mathrm{~kg}^{-1} \mathrm{~K}^{-1}$ \\
$g_{h}$ & 0.01 & $\mathrm{~m} \mathrm{~s}-1$ \\
$g_{e}$ & 0.01 & $\mathrm{~m} \mathrm{~s}^{-1}$ \\
$\beta$ & 0.2 & - \\
$\gamma_{\vartheta}$ & $4.78 \times 10^{-3}$ & $\mathrm{~K} \mathrm{~m}^{-1}$ \\
$\gamma_{q}$ & $-0.00145 \times 10^{-3}$ & $\mathrm{~kg} \mathrm{~kg}^{-1} \mathrm{~m}^{-1}$ \\
$\vartheta_{f_{0}}$ & 288 & $\mathrm{~K}$ \\
$q_{f_{0}}$ & 0.00785 & $\mathrm{~kg} \mathrm{~kg}^{-1}$ \\
$t_{0}$ & 6 & $\mathrm{hours}$ \\
$Q_{m}$ & 400 & $\mathrm{~W} \mathrm{~m}^{-2}$ \\
\hline
\end{tabular}

data from Davies and Allen (1973) show Bowen ratios typically between 0.3 and 0.1 , for temperatures increasing from $\approx 15^{\circ} \mathrm{C}$ to $\approx 25^{\circ} \mathrm{C}$. Since also $\lambda$ is almost constant at those temperatures, it means that (21) can be approximated as

$$
\frac{d \tilde{q}}{d \tilde{\theta}}=\varphi-\frac{\tilde{q}}{\tilde{\theta}}
$$

where $\varphi$ is a constant equal to

$$
\varphi=\frac{c_{p}}{\lambda(1+\beta) \mathrm{Bo}_{0}}+\frac{(1+2 \beta) \gamma_{q}}{(1+\beta) \gamma_{\vartheta}} .
$$

Note that the first term of the right-hand side of (23) is typically positive, while the second term tends to be negative due to the fact that $\gamma_{q}$ is typically small but lower than zero (in the computations we use a typical value of $-0.00145 \times 10^{-3} \mathrm{~kg} \mathrm{~kg}^{-1} \mathrm{~m}^{-1}$ as in Lhomme et al. (1998), see Table 1).

The solution of (22) is now easily found as,

$$
\tilde{q}=\frac{\varphi}{2} \tilde{\vartheta}+\frac{C_{2}}{\tilde{\vartheta}}
$$

where, as usual, we neglect the morning transition and set $C_{2}=0$. As a result, we obtain a linear relation of the mixed-layer specific humidity with temperature analogous to (15). Because of (8), a linear relationship also links specific humidity and height as

$$
\tilde{q}=\gamma_{q}^{\prime} h,
$$

where we have introduced a 'lapse rate' for the humidity at the top of the mixed layer. Using (23), (24) and (8), the latter results in a linear combination of the lapse rates of the free-atmosphere profiles,

$$
\gamma_{q}^{\prime}=\frac{1}{2}\left[\gamma_{\vartheta} \frac{c_{p}}{\lambda(1+2 \beta) \mathrm{Bo}}+\gamma_{q}\right]
$$

where the first term is the contribution due to surface evaporation and the second term results from the entrainment from the free atmosphere.

It is interesting to analyze the sign and value of the coefficient $\varphi$ (which is also the same as that of $\gamma_{q}^{\prime}$ ) as a function of the Bowen ratio for a typical value of $\gamma_{q}$ (Table 1). As seen in Fig. 3 (left panel), for well-watered conditions (e.g., Bo $\approx 0.1-0.3) \varphi$ is positive, which implies that the specific humidity of the mixed layer increases during the day. However, as 

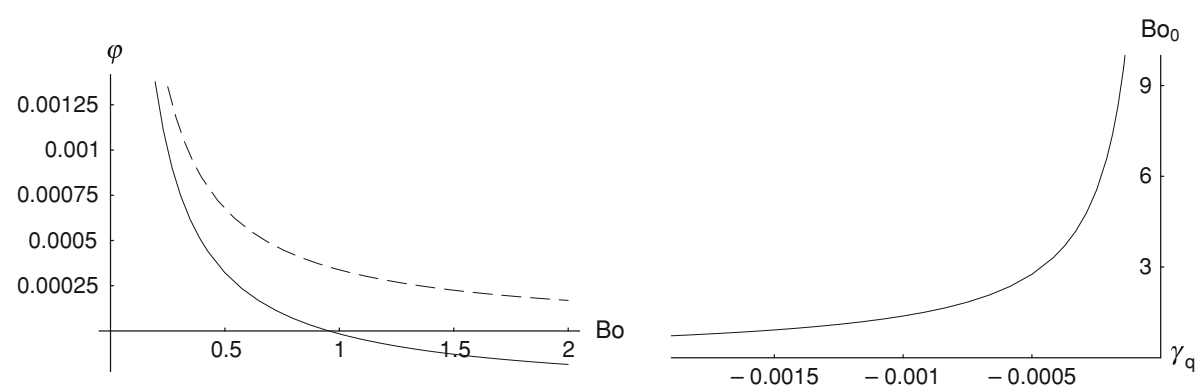

Fig. 3 Left Behaviour of the constant $\varphi$ as a function of the Bowen ratio. Continuous line refers to $\gamma_{q}=-0.00145 \times 10^{-3} \mathrm{~kg} \mathrm{~kg}^{-1} \mathrm{~m}^{-1}$, while dashed line is for $\gamma_{q}=0$ (see Table 1 for other parameter values). Right Behaviour of the Bowen ratio $\mathrm{Bo}_{0}$ that maintains constant mixed-layer specific humidity as a function of the mixed-layer specific humidity 'lapse rate'
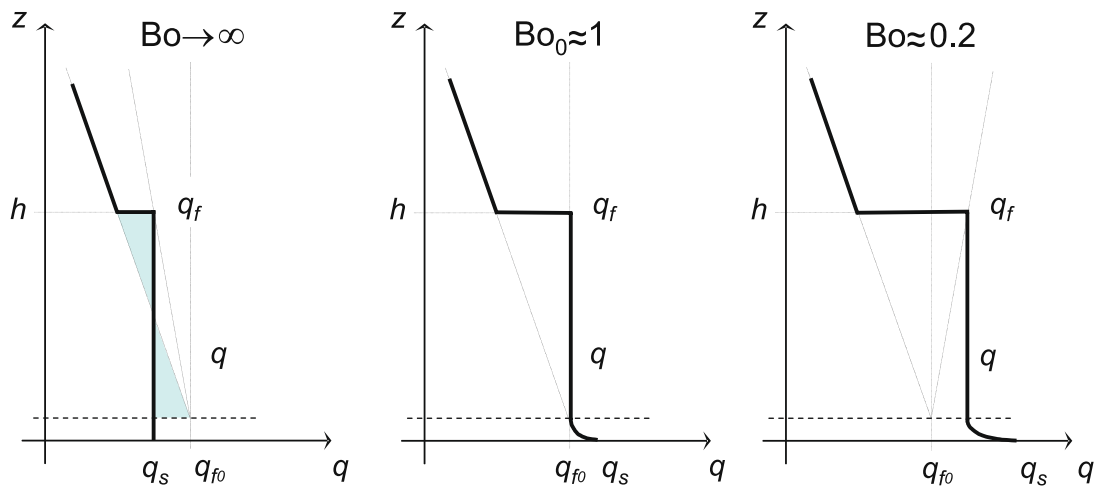

Fig. 4 Types of specific humidity profiles for three different values of the Bowen ratio

the Bowen ratio increases the input of moisture is reduced along with the values of $\varphi$ and $\gamma_{q}^{\prime}$, until they become zero when

$$
\mathrm{Bo}_{0}=-\frac{c_{p} \gamma_{\vartheta}}{\lambda(1+2 \beta) \gamma_{q}}
$$

Using typical values of the parameters (see Table 1 ), this happens for $\mathrm{Bo}_{0} \approx 1$ (Fig. 3, left panel). Note that the above expression is valid only for $\gamma_{q}<0$, while for $\gamma_{q} \geq 0$ the specific humidity always increases as both $\varphi$ and $\gamma_{q}^{\prime}$ are always positive (Fig. 3, right panel shows the Bowen ratios $\mathrm{Bo}_{0}$ that ensure constant specific humidity as a function of $\gamma_{q}$ ).

For the limiting case of Bo $\rightarrow \infty$, which takes place for a dry surface (e.g., $E \rightarrow 0$ or $g_{e} / g_{h}=0$ ), the lapse rate becomes one half that of the free atmosphere, $\gamma_{q}^{\prime}=1 / 2 \gamma_{q}$ (note that this is typically a negative value). The reason for this is that the boundary layer does not receive any moisture from the surface, while it entrains drier air from the free atmosphere above. The growth of the discontinuity, schematically depicted in Fig. 4, is such to maintain the two shaded areas equal and thus ensure moisture conservation. This is similar to the temperature growth in conditions of zero sensible heat flow at the surface (see Fig. 2 for $\beta \rightarrow \infty)$.

Although approximated, the assumption of constant Bowen ratio gives simple relations among the mixed layer variables. Section 6 presents the temporal evolution of the ABL following this assumption. 


\section{Limiting Behaviours of the Bowen Ratio}

The reason for the Bowen ratio to remain almost constant during mid-day hours is explained by the limiting behaviour of the Bowen ratio as a function of the net available energy $Q$. We begin by re-writing (4), and separating the contributions due to temperature differences between the surface and the atmosphere from that due to the saturation deficit of the air, as typically done in the Penman-Monteith or combination approach (Raupach 2001; Brutsaert 2005),

$$
E=g_{e} \rho\left(q_{s}^{*}-q^{*}+q^{*}-q\right)=g_{e} \rho\left[\epsilon_{r}\left(\vartheta_{s}-\vartheta\right)+\left(q^{*}-q\right)\right],
$$

where $\epsilon_{r}$ is the slope of the saturated specific humidity curve computed at a suitable reference temperature (comprised between the surface and the atmospheric temperature). As noted in Raupach (2001), this is simply an algebraic manipulation that does not entail any approximation. Dividing (28) by $g_{e} \rho\left(\vartheta_{s}-\vartheta\right)$ it follows that

$$
\frac{q_{s}^{*}-q}{\vartheta_{s}-\vartheta}=\epsilon_{r}+\frac{q^{*}-q}{\vartheta_{s}-\vartheta} .
$$

Moreover, combining (28) and (5) and substituting into (1) it is possible to solve for

$$
\vartheta_{s}=\vartheta+\frac{Q / \rho-\lambda g_{e}\left(q^{*}-q\right)}{\lambda g_{e} \epsilon_{r}+g_{h} c_{p}},
$$

from which

$$
\frac{q_{s}^{*}-q}{\vartheta_{s}-\vartheta}=\epsilon_{r}+\frac{\left(\lambda g_{e} \epsilon_{r}+g_{h} c_{p}\right)\left(q^{*}-q\right)}{Q / \rho-\lambda g_{e}\left(q^{*}-q\right)} .
$$

The latter can be introduced into (19) to obtain a relationship between the Bowen ratio and the net available energy.

Two limiting behaviours can be readily analysed: one valid early in the morning and evening, when $Q$ is very small, which gives

$$
\left.\frac{\tilde{q}_{s}^{*}-\tilde{q}}{\tilde{\vartheta}_{s}-\tilde{\vartheta}}\right|_{Q \rightarrow 0}=-\frac{g_{h} c_{p}}{g_{e} \lambda},
$$

and the other, when $Q$ is very large, whereby

$$
\left.\frac{\tilde{q}_{s}^{*}-\tilde{q}}{\tilde{\vartheta}_{s}-\tilde{\vartheta}}\right|_{Q \rightarrow \infty}=\epsilon_{r} .
$$

The corresponding Bowen ratios are, respectively,

$$
\operatorname{Bo}_{Q \rightarrow 0}=-1
$$

and

$$
\operatorname{Bo}_{Q \rightarrow \infty}=\frac{g_{h} c_{p}}{g_{e} \lambda \epsilon_{r}} .
$$

The first case for $Q \rightarrow 0$ is easily understood in terms of the energy balance (1) with no net energy input, where the evaporation that cools the surface is balanced by an opposite sensible heat flux. This of course stems from the simplified form of (1) that assumes steady-state conditions (i.e., no heat storage) of the surface energy balance. More interesting is the behaviour when $Q$ is very large, which shows that the Bowen ratio is simply given in terms of the conductance ratio and thermodynamic quantities linked to the saturated vapour pressure 

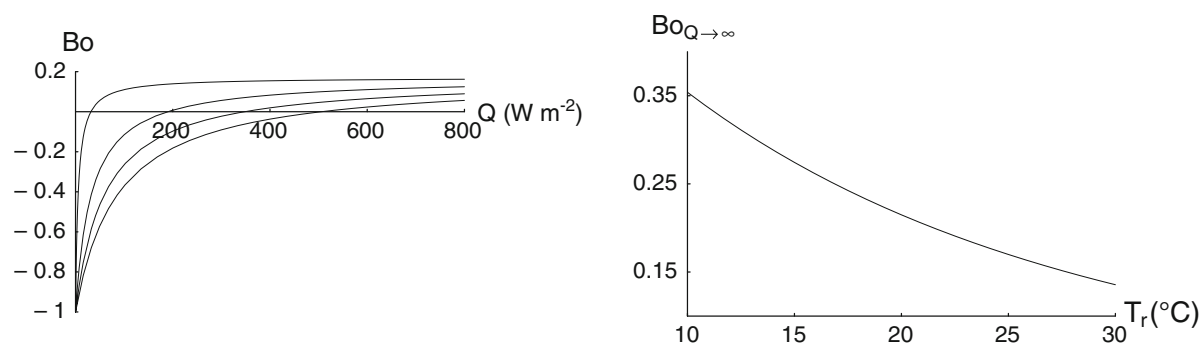

Fig. 5 Left Bowen ratio as a function of the net available energy for different values of the vapour saturation deficit (from top to bottom, lines refer to $q^{*}-q=0.001,0.006,0.011,0.016$, respectively) for a reference temperature of $25^{\circ} \mathrm{C}$. Right Limiting value of the Bowen ratio for very large net available energy as a function of the reference temperature introduced in (28) (see Table 1 for other parameter values)

curve. It is also noteworthy that the limiting value is the same as that obtained in conditions of potential evaporation, i.e., when $\tilde{q}^{*}-\tilde{q}=0$, independently of the value of $Q$.

In either limiting case, the Bowen ratio becomes almost independent of the atmospheric conditions. As shown in Fig. 5 (left panel) the lower the pressure deficit of the atmosphere, the faster (in terms of $Q$ ) this decoupling from the atmospheric conditions is achieved. For high enough $Q$ the range of variation of the Bowen ratio is rather limited, thus explaining the almost-constant values observed during midday hours. In contrast, for low and intermediate values of $Q$ and unsaturated conditions, the Bowen ratio is more sensitive to the feedback from the atmosphere. It is also interesting to note that for large $Q$ the limiting value of the Bowen ratio does not vary strongly with temperature (see Fig. 5, right panel), but remains dependent on the ratio of the surface conductances (e.g., water stress).

\section{Diurnal Evolution}

The approximate solutions assuming constant Bowen ratio and neglecting the morning transition are extremely simple and can be used to obtain analytically the diurnal evolution of the main ABL variables. Using a simple parabolic behaviour of the available energy

$$
Q(t)=Q_{m}\left[1-\left(t / t_{0}-1\right)^{2}\right],
$$

with $0<t<2 t_{0}$, and $H=Q \mathrm{Bo} /(1+\mathrm{Bo})$, equation (10) can be integrated to give the diurnal evolution of the ABL height,

$$
h=\left[\frac{2}{3} \frac{(1+2 \beta) \operatorname{Boo}\left(3 t_{0}-t\right) t^{2}}{\rho c_{p} \gamma_{\vartheta}(1+\mathrm{Bo}) t_{0}^{2}}\right]^{\frac{1}{2}} .
$$

Figures 6 and 7 show the ensuing diurnal evolutions of the main ABL variables, obtained using (37), (15) and (25), while Fig. 8 gives the final values of the ABL height and relative humidity at sunset $\left(t=2 t_{0}\right)$ as a function of the values of the Bowen ratio. Note how the behaviour of the relative humidity is more complex (Ek and Mahrt 1994) than the other variables and quite sensitive to the values of the Bowen ratio and the atmospheric conditions during the early morning hours when $Q$ is still low. This behaviour of the diurnal evolution is expected to be quite realistic because the constant Bowen ratio approximation is acceptable during the central part of the day when the available energy is higher (Fig. 5, left panel), while early and late in the day, the energy fluxes are small and thus the errors due to the 

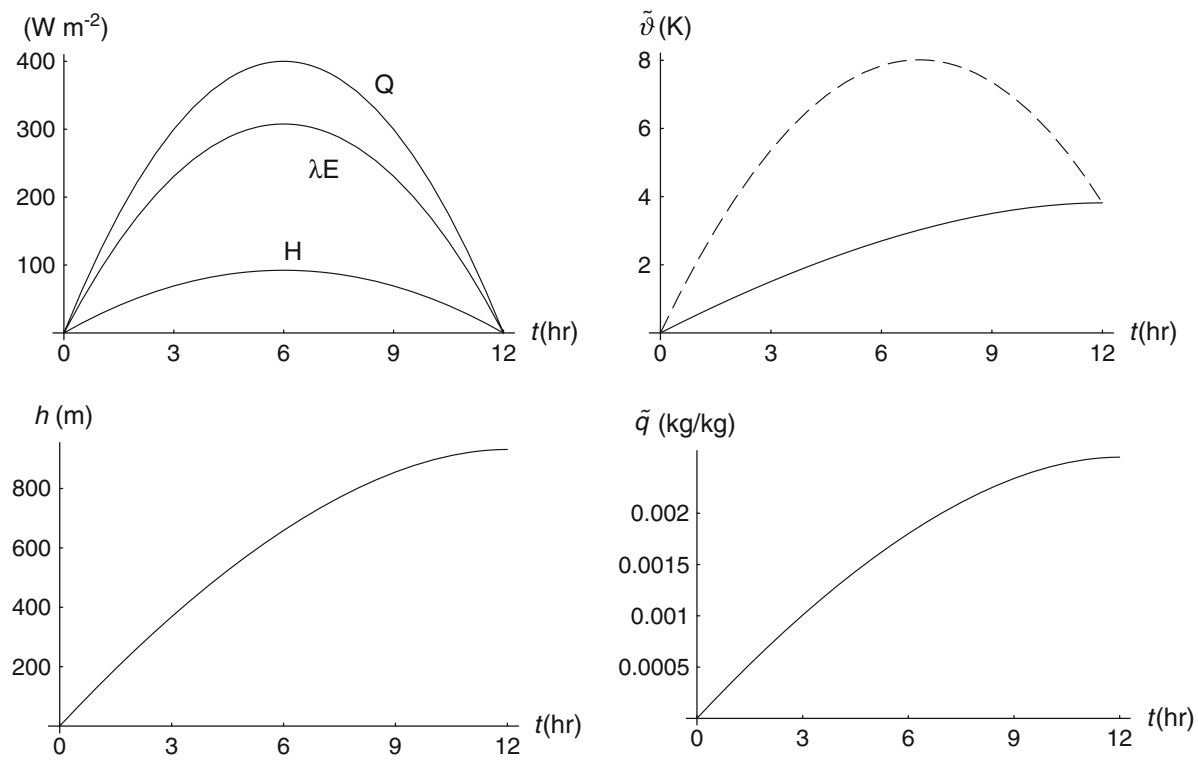

Fig. 6 Diurnal trends of the main ABL variables obtained assuming constant Bowen ratio and parabolic behaviour of available energy (see Table 1 for parameter values). Dashed line is surface temperature

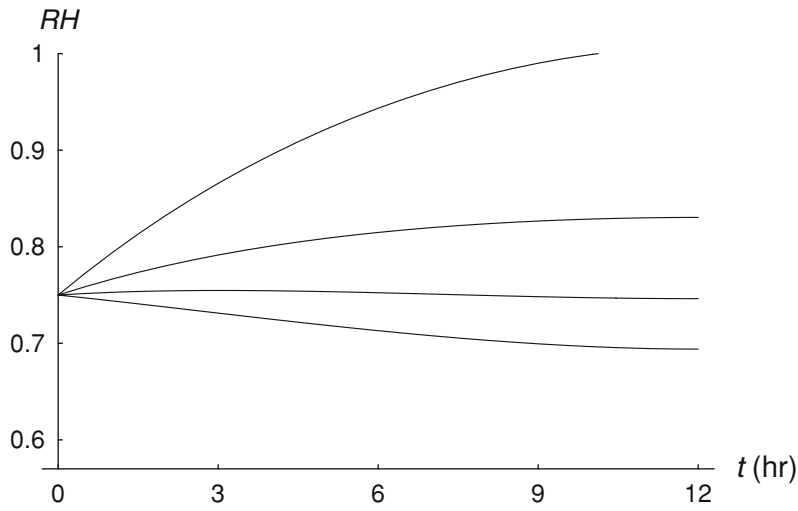

Fig. 7 Diurnal trend of relative humidity for different values of the Bowen ratio. From top to bottom the curves refer to $\mathrm{Bo}=0.1,0.2,0.3,0.4$ (see Table 1 for parameter values)

approximation should not play too important a role. Future work will analyze in detail the entity of these errors and the role of the initial conditions during the morning transition.

\section{Conclusions}

The dynamics of the ABL are described by the coupled nonlinear ordinary differential equations (6), (7) and (10), and the algebraic equations (1), (4) and (5). After a relatively complex initial transition (Tennekes 1973), the ABL evolves, driven by high levels of available energy, along a relatively simple trajectory characterized by an almost-constant value of the 

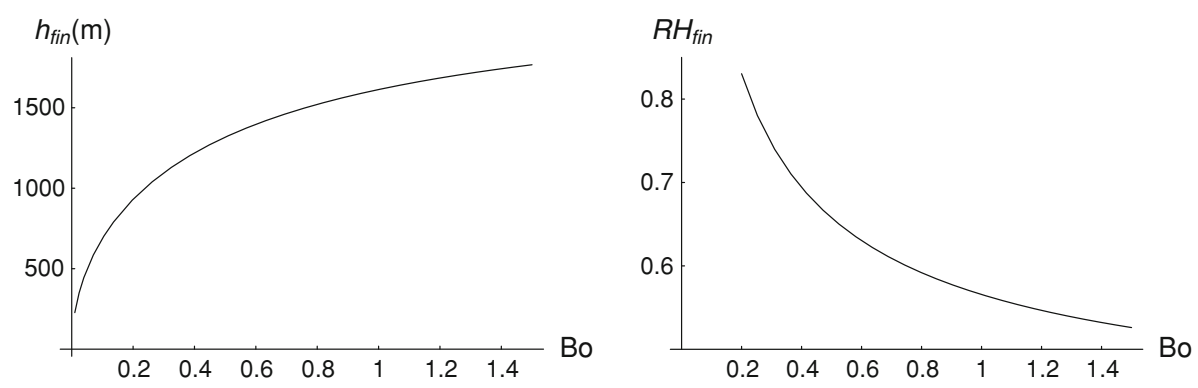

Fig. 8 Height of the ABL and relative humidity at sunset as a function of the Bowen ratio (see Table 1 for parameter values)

Bowen ratio. In these midday conditions the values of the Bowen ratio tend to be close to the $Q \rightarrow \infty$ limit, which coincides with the potential evaporation limit (e.g., Raupach 2000, 2001; Brutsaert 2005) and depends only on the relative value of the conductances to latent and sensible heat fluxes and on thermodynamic quantities. At both very low and very high energy levels the Bowen ratio tends to become independent of the atmospheric conditions, apart from a relatively weak dependence on temperature for the very large $Q$ limit.

In the approximation of constant Bowen ratio, the relationships among the main ABL variables and the jumps in temperature and specific humidity at the inversion layer atop the $\mathrm{ABL}$, are linear. The analytical solutions of the time evolution of the ABL obtained under constant Bowen ratio from the linear relations (15) and (25) are simple enough and may be useful as benchmarks for more complex models.

\section{Appendix: Justification of Equation (10)}

Equation (10) can be justified using arguments due to Tennekes (1973), Betts (1973), and Garratt (1992), who noticed that the potential temperature inversion, $\vartheta_{f}-\vartheta=\delta$, tends to grow linearly with the height of the ABL. We present here the steps for completeness and to point out the fact that the approximations leading to (10) require neglecting the effect of the initial conditions (i.e., the morning transition).

The surface sensible heat flux is Brutsaert (2005)

$$
H=\rho c_{p}\left(\overline{\vartheta^{\prime} w^{\prime}}\right)_{s},
$$

where primes denote turbulence fluctuations around the mean. At the top of the mixed layer, the turbulent flux is linked to the entrainment of potential temperature. As the ABL grows, thus

$$
\delta \frac{d h}{d t}=-\left(\overline{\vartheta^{\prime} w^{\prime}}\right)_{h}
$$

coupled to the kinetic equation for the inversion strength (Tennekes 1973)

$$
\frac{d \delta}{d t}=\gamma_{\vartheta} \frac{d h}{d t}-\frac{d \vartheta}{d t} .
$$

The energy equation for the well-mixed layer is

$$
h \frac{d \vartheta}{d t}=\left(\overline{\vartheta^{\prime} w^{\prime}}\right)_{s}-\left(\overline{\vartheta^{\prime} w^{\prime}}\right)_{h}
$$

which, with the substitutions of (38) and (39), becomes Eq. (6). 
The turbulent flux at the top of the ABL can be approximated using the turbulence energy balance equation at the inversion. Following Tennekes (1973) a good approximation is given by the interpolation relation

$$
-\left(\overline{\vartheta^{\prime} w^{\prime}}\right)_{h}=A \frac{T_{0} u_{*}^{3}}{g h}+\beta\left(\overline{\vartheta^{\prime} w^{\prime}}\right)_{s},
$$

where the first term on the right-hand side is the flux due to growth of the ABL by mechanical turbulence while the second is due to the buoyancy-generated turbulence. In the previous equation, $g / T_{0}$ is the buoyancy parameter, $u_{*}$ is the surface friction velocity and $A$ and $\beta$ are parameters. $A$ is typically 2.5 and $\beta$ lies between 0.1 and 0.4 with a typical value of 0.2. Since this second term is dominant after the early morning transition (Tennekes and Driedonks 1981; Driedonks and Tennekes 1984), we further approximate, as is customary,

$$
\left(\overline{\vartheta^{\prime} w^{\prime}}\right)_{h}=-\beta\left(\overline{\vartheta^{\prime} w^{\prime}}\right)_{s}
$$

The previous equations describe the growth of the ABL height $(h)$ and the inversion depth $(\delta)$, once the surface flux $\left(H\right.$ or $\left.\left(\overline{\vartheta^{\prime} w^{\prime}}\right)_{s}\right)$ is known either from measurements or from solving the surface energy balance and computing the sensible heat flux from (1), (4) and (5).

Further simplifications are possible if one neglects the effect of the initial conditions during the morning transition. Substitution (39) and (41) into (40) gives

$$
\frac{d}{d t}(\delta h)=\gamma_{\vartheta} h \frac{d h}{d t}-\left(\overline{\vartheta^{\prime} w^{\prime}}\right)_{s}
$$

which, combined with (43), provides a differential equation for $\delta$ and $h$ implicit in time (Tennekes 1973)

$$
h \frac{d \delta}{d h}+\left(\frac{1}{\beta}+1\right) \delta-\gamma_{\vartheta} h=0,
$$

the solution of which is

$$
\delta=\frac{\gamma_{\vartheta} \beta}{2+\beta} h+C_{3} h^{\frac{\beta+1}{\beta}}
$$

With $\delta=0$ for $h=0$ (no morning transition), the previous relation becomes

$$
\delta=\frac{\gamma_{\vartheta} \beta}{2+\beta} h
$$

which is a more general form of Eq. 29 of Tennekes (1973). Substituting now (47) in (44) allows us to write

$$
\gamma_{\vartheta} h \frac{d h}{d t}-\gamma_{\vartheta} \frac{\beta}{1+2 \beta} 2 h \frac{d h}{d t}=-\left(\overline{\vartheta^{\prime} w^{\prime}}\right)_{s} .
$$

Using (38), this readily gives (10), which is also Eq. 6.18 of Garratt (1992).

Acknowledgements Stimulating and enjoyable discussions with Wilfried Brutsaert and Marc Parlange are gratefully acknowledged. Comments by Mike Raupach, Giulia Vico and two anonymous reviewers helped improve the paper. This research was supported in part by the US National Science Foundation under grants EAR-0628432 and EAR-0635787 and by a collaboration grant from the US Department of Agriculture, Agricultural Research Service, Temple, TX. The support of the Landolt \& Cie visiting chair "Innovative strategies for a sustainable future" at the École Polytechnique Fédérale de Lausanne (EPFL), Lausanne, Switzerland is also gratefully acknowledged. 


\section{References}

Betts A (1973) Non-precipitating cumulus convection and its parameterization. Q J Roy Meteorol Soc 99:178-196

Bowen I (1926) The ratio of heat losses by conduction and by evaporation from any water surface. Phys Rev 27:779-787

Brutsaert W (1987) Nearly steady convection and the boundary-layer budgets of water vapor and sensible heat flux. Boundary-Layer Meteorol 39:283-300

Brutsaert W (2005) Hydrology: an introduction. Cambridge University Press, Cambridge, 605 pp

Brutsaert W, Chen D (1996) Diurnal variation of surface fluxes during thorough drying (or severe drought) of natural prairie. Water Resour Res 32:2013-2019

Brutsaert W, Sugita M (1992) Application of self-preservation in the diurnal evolution of the surface energy budget to determine daily evaporation. J Geophys Res 97(D17):18377-18382

Carson D (1973) The development of a dry inversion-capped convectively unstable boundary layer. Q J Roy Meteorol Soc 99:450-467

Daly E, Porporato A, Rodriguez-Iturbe I (2004) Modeling photosynthesis, transpiration, and soil water balance: hourly dynamics during interstorm periods. J Hydromet 5:546-558

Davies J, Allen C (1973) Equilibrium, potential and actual evaporation from cropped surfaces in southern Ontario. J Appl Meteorol 12:649-657

de Bruin H (1983) A model for the Pristley-Taylor $\alpha$. J Clim Appl Meteorol 22:572-578

Driedonks A (1982) Sensitivity analysis of the equations for a convective mixed layer. Boundary-Layer Meteorol 22:475-480

Driedonks A, Tennekes H (1984) Entrainment effects in the well-mixed atmospheric boundary layer. Boundary-Layer Meteorol 30:75-105

Ek M, Holtslag A (2004) Influence of soil moisture on boundary layer cloud development. J Hydrometeorol 5:86-99

Ek M, Mahrt L (1994) Daytime evolution of relative humidity at the boundary layer top. Mon Weather Rev 122:2709-2721

Garratt J (1992) The atmospheric boundary layer. Cambridge University Press, UK, 316 pp

Juang J-Y, Porporato A, Stoy P, Siqueira M, Oishi A, Detto M, Kim H-S, Katul G (2007) Hydrologic and atmospheric controls on initiation of convective precipitation. Water Resour Res 43:W03421

Lhomme J, Elguero E, Chehbouni A, Boulet G (1998) Stomatal control of transpiration: examination of Monteith's formulation of canopy resistance. Water Resour Res 34:2301-2308

McNaughton K, Spriggs T (1986) A mixed-layer model for regional evaporation. Boundary-Layer Meteorol 34:243-262

Raupach M (1991) Vegetation-atmosphere interactions in homogeneous and heterogeneous terrain: some implications of mixed-layer dynamics. Vegetatio 91:105-120

Raupach M (1998) Radiative physiological, aerodynamic and boundary-layer feedbacks on the terrestrial surface energy balance. Glob Change Biol 4:477-494

Raupach M (2000) Equilibrium evaporation and the convective boundary layer. Boundary-Layer Meteorol 96:107-141

Raupach M (2001) Combination theory and equilibrium evaporation. Q J Roy Meteorol Soc 127:1149-1181

Siqueira M, Porporato A, Katul G (2009) Soil moisture feedbacks on convection triggers: the role of soil-plant hydrodynamics. J Hydrometeorol 10:96-112

Stewart J, Thom A (1973) Energy budgets in pine forest. Q J Roy Meteorol Soc 99:154-170

Tennekes H (1973) A model for the dynamics of the inversion above a convective boundary layer. J Atmos Sci 30:558-567

Tennekes H, Driedonks A (1981) Basic entrainment equations for the atmospheric boundary layer. BoundaryLayer Meteorol 20:515-531 